
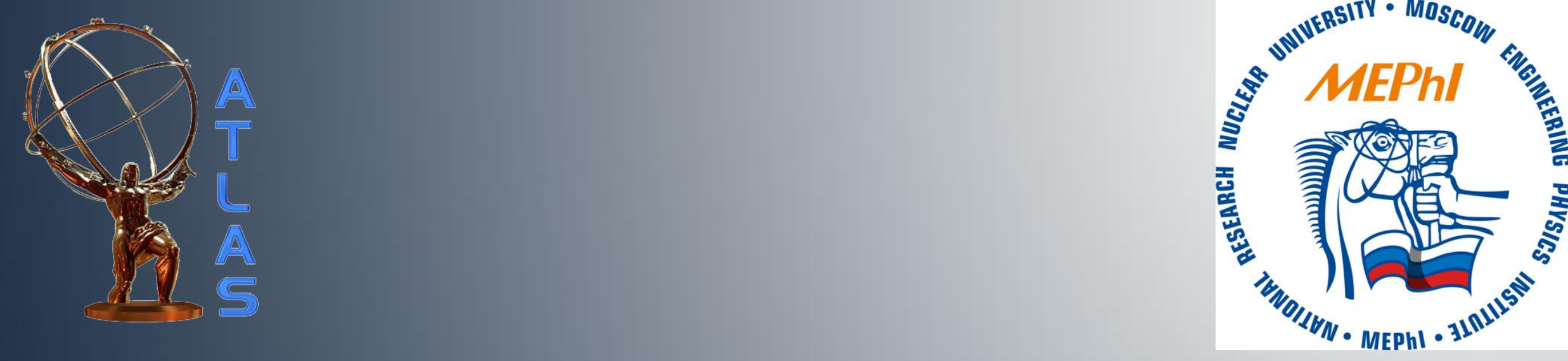

\title{
Electroweak measurements with the ATLAS detector
}

Krasnopevtsev D. on behalf of the ATLAS Collaboration Moscow NRNU MEPhI

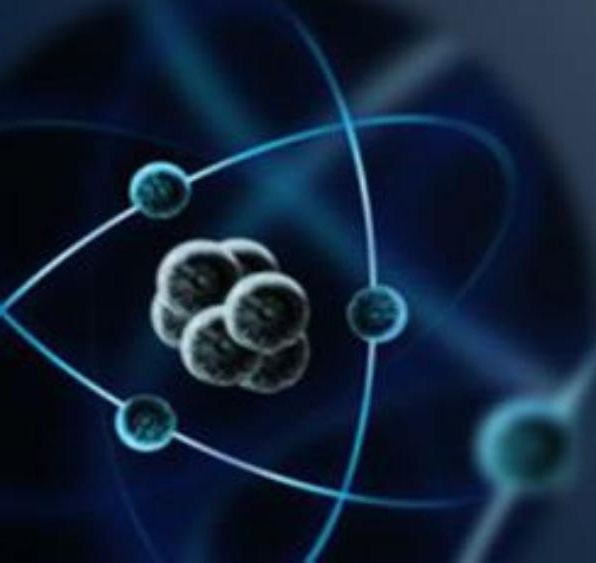




\section{Outline}

Standard Model (SM) electroweak measurements aim to:

$>$ validate SM in new energy scale

$>$ improve accuracy of known SM parameters

$>$ estimate new physics contributions

This talk is devoted to Run 1,8 TeV studies:

SM production cross section measurements

WW production

$>$ Inclusive 4l production

$>$ Vector boson fusion Z

$>$ Vector boson scattering WW

$>$ Wyvproduction

\section{ATLAS}

-Preliminary

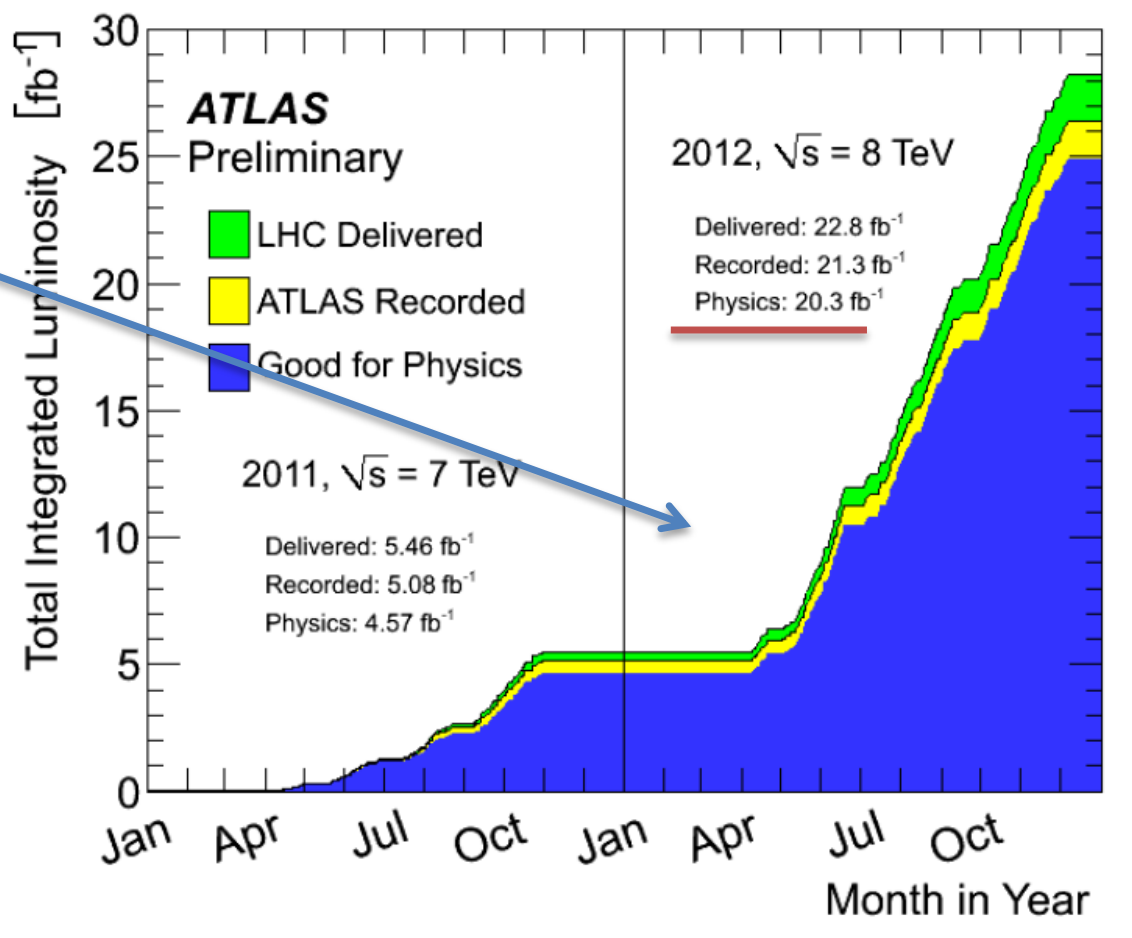




\section{Standard Model Production Cross Section Measurements}

$\frac{\pi}{6}$

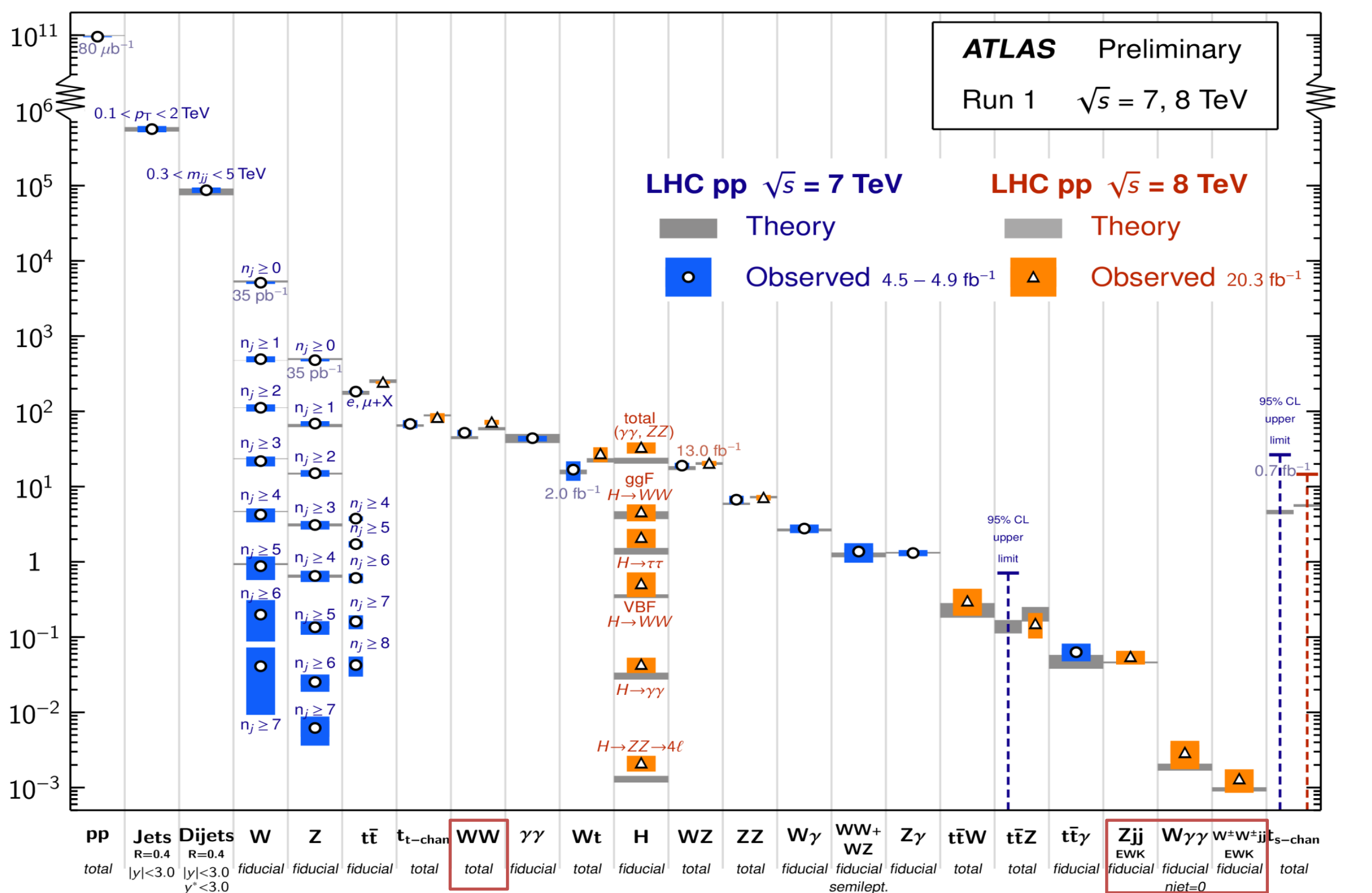

+41 production and Recent run 2 results 


\section{WW production}

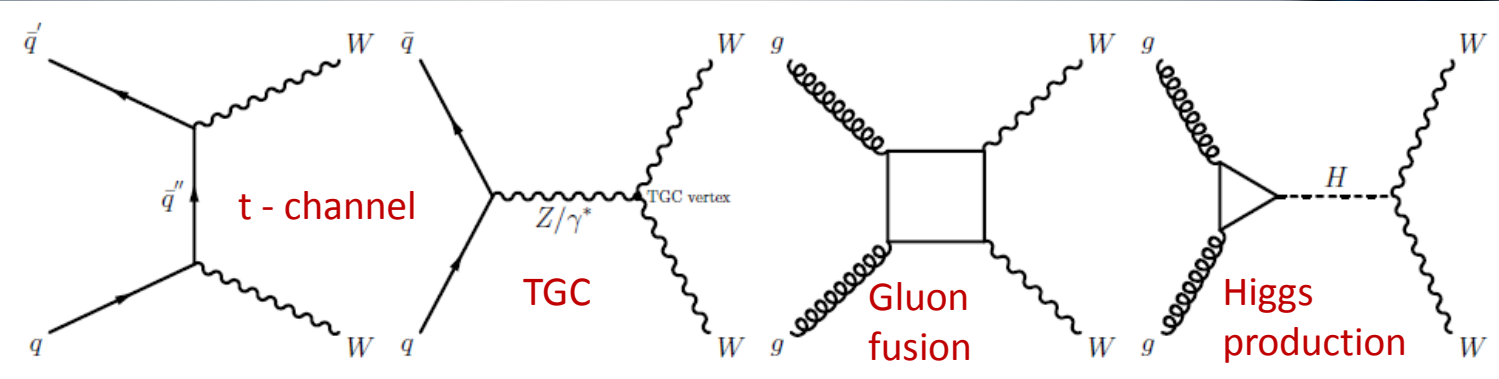

$>$ Important test of the non-Abelian structure of SM

$>$ Cross section measurements are sensitive to

ATLAS-CONF-2014-033 anomalous triple gauge couplings (aTGC)

$>$ Non-resonant WW production is an irreducible background process to Higgs boson studies

$>$ Signature

- two isolated leptons

- high missing energy (MET)

$>$ Dominant backgrounds

\begin{tabular}{lccc}
\hline \hline Channel & $e^{ \pm} \mu^{\mp}$ & $e^{+} e^{-}$ & $\mu^{+} \mu^{-}$ \\
\hline Observed Events & 5067 & 594 & 975 \\
\hline Total expected events & $4376 \pm 26 \pm 280$ & $536 \pm 10 \pm 42$ & $873 \pm 12 \pm 63$ \\
\hline MC $W W$ signal & $3224 \pm 10 \pm 248$ & $346 \pm 3 \pm 32$ & $610 \pm 5 \pm 56$ \\
\hline Top(data-driven) & $609 \pm 18 \pm 52$ & $92 \pm 7 \pm 8$ & $127 \pm 9 \pm 11$ \\
W+jets(data-driven) & $220 \pm 15 \pm 112$ & $14 \pm 5 \pm 9$ & $3 \pm 5 \pm 6$ \\
Z+jets (data-driven) & $166 \pm 3 \pm 26$ & $55 \pm 1 \pm 23$ & $96 \pm 2 \pm 27$ \\
Other dibosons (MC) & $157 \pm 4 \pm 31$ & $30 \pm 2 \pm 5$ & $39 \pm 1 \pm 5$ \\
\hline Total background & $1152 \pm 24 \pm 130$ & $190 \pm 9 \pm 26$ & $264 \pm 11 \pm 30$ \\
\hline \hline
\end{tabular}

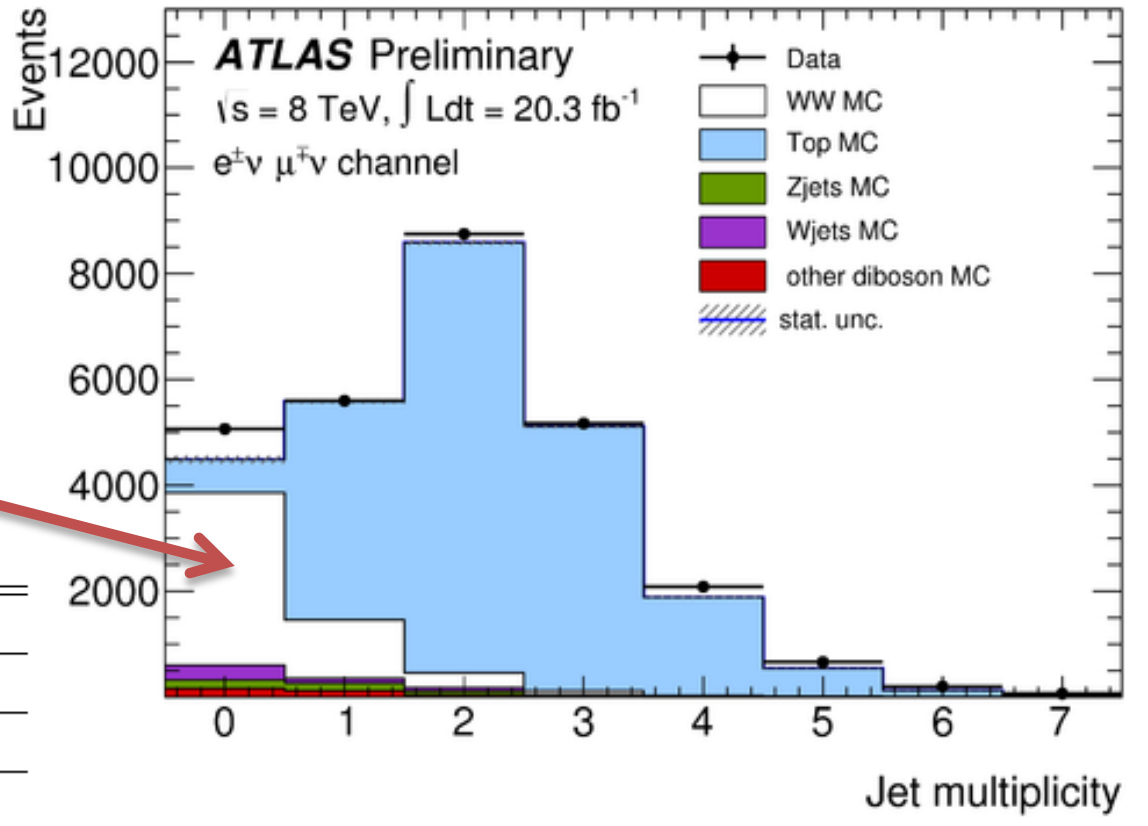




\section{WW results}

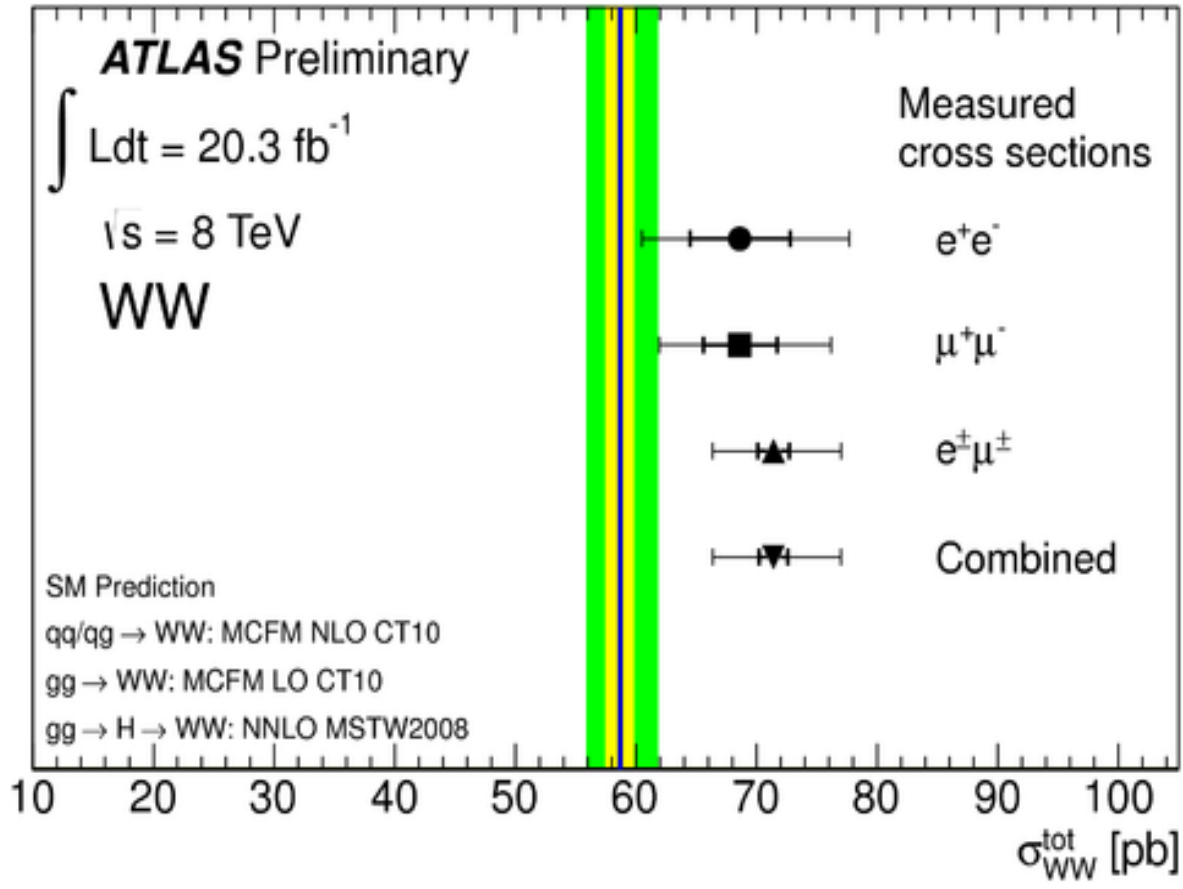

Fiducial cross sections:
$>$ The individual channels are compatible within their uncertainties.

$>$ The measured combined cross section differs from the partial NNLO SM prediction computed using CT10 PDF by $+2.1 \sigma$ using the standard PDF and scale uncertainties.

Meanwhile

$>$ Compatible with full NNLO prediction at about $1 \sigma$

New result from CMS (arXiv:1507.03268) Measured total $\sigma$ agree well with NNLO prediction.

$\sigma_{e \mu}^{\text {fid }} 377.8_{-6.8}^{+6.9}$ (stat) ${ }_{-22.2}^{+25.1}$ (syst) ${ }_{-10.7}^{+11.4}$ (lumi) fb

Compatible with approximate NNLO+NNLL calculation

$357.9 \pm 14.4 \mathrm{fb}$

arXiv: 1410.4745 


\section{1 inclusive}

Goal

$>$ To test the validity of the Standard Model through the interplay of QCD and Electroweak effects for different 4 l production mechanisms.

\section{4l production at the $\mathrm{LHC}$}

- qq-originated $4 \mathrm{l}$ production

- 4 I from Higgs boson decays

- Non-resonant ZZ $\rightarrow$ 4l production via ggF, VBF and VBS processes

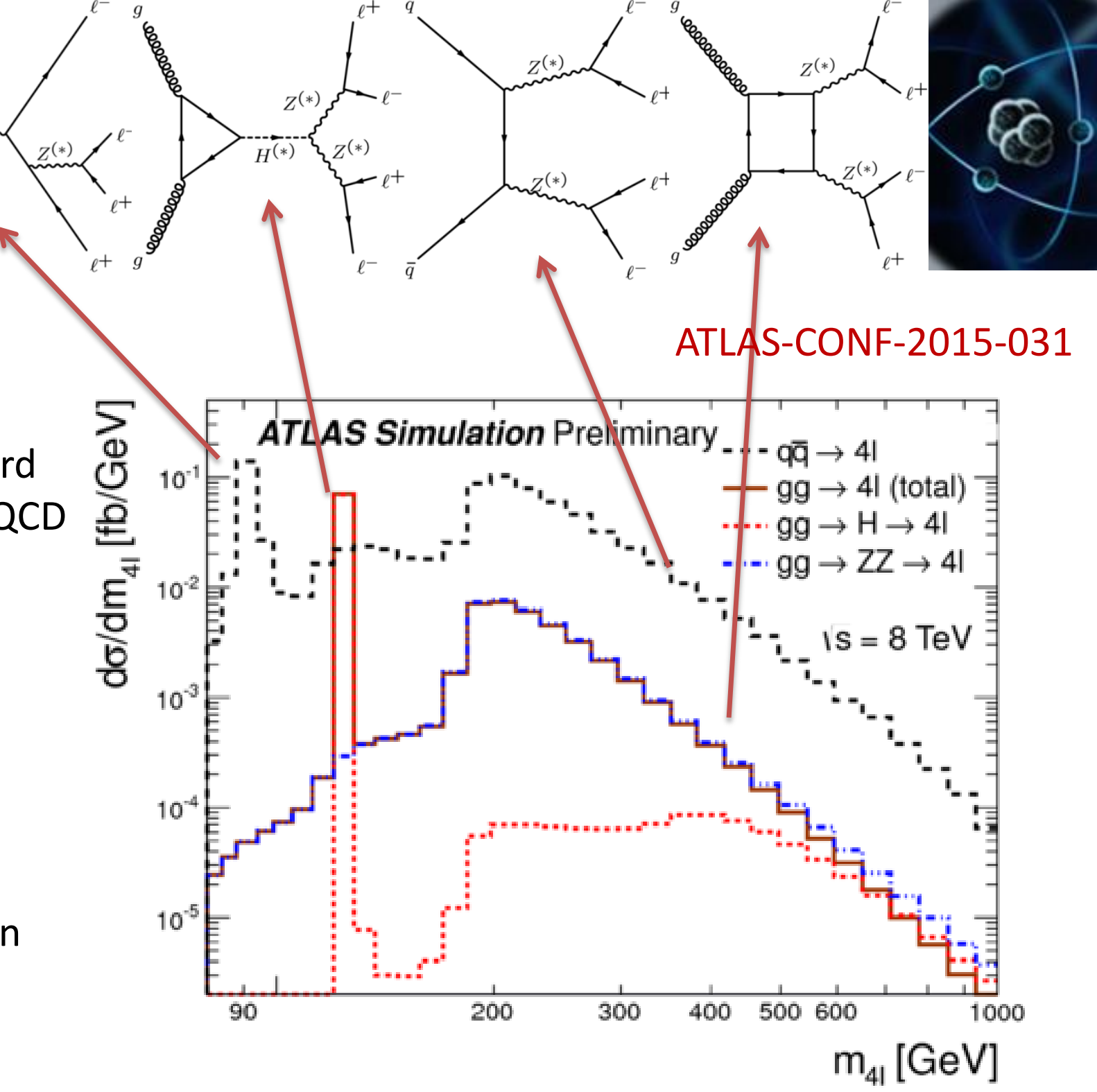




\section{1 inclusive}

\section{Event yield}

Selection (leptonic channel is clean)

\begin{tabular}{ccccccc}
\hline Channel & $N^{\text {Data }}$ & $N_{\text {expected }}^{\text {Total }}$ & $N_{\text {non-gg }}^{\text {signal }}$ & $N_{g g}^{\text {signal }}$ & $N_{\tau}^{M C}$ & $N_{b k g}$ \\
\hline $4 e$ & 85 & $80 \pm 4$ & $68.4 \pm 3.4$ & $6.24 \pm 0.31$ & $1.28 \pm 0.06$ & $3.6 \pm 0.5$ \\
$4 \mu$ & 156 & $150.2 \pm 2.9$ & $128.2 \pm 2.5$ & $11.00 \pm 0.21$ & $2.18 \pm 0.09$ & $9.0 \pm 1.5$ \\
$2 e 2 \mu$ & 235 & $205 \pm 5$ & $172 \pm 5$ & $16.0 \pm 0.4$ & $3.08 \pm 0.13$ & $13.6 \pm 2.1$ \\
\hline Total & 476 & $435 \pm 9$ & $369 \pm 9$ & $33.3 \pm 0.8$ & $6.54 \pm 0.14$ & $26.2 \pm 3.6$ \\
\hline
\end{tabular}

- The overall background estimation uncertainty is $12 \%$ (the impact on the measured cross sections is $<1 \%$ ).

Generally good agreement

Small excess over the best available theory predictions (could be explained by statistical fluctuations)

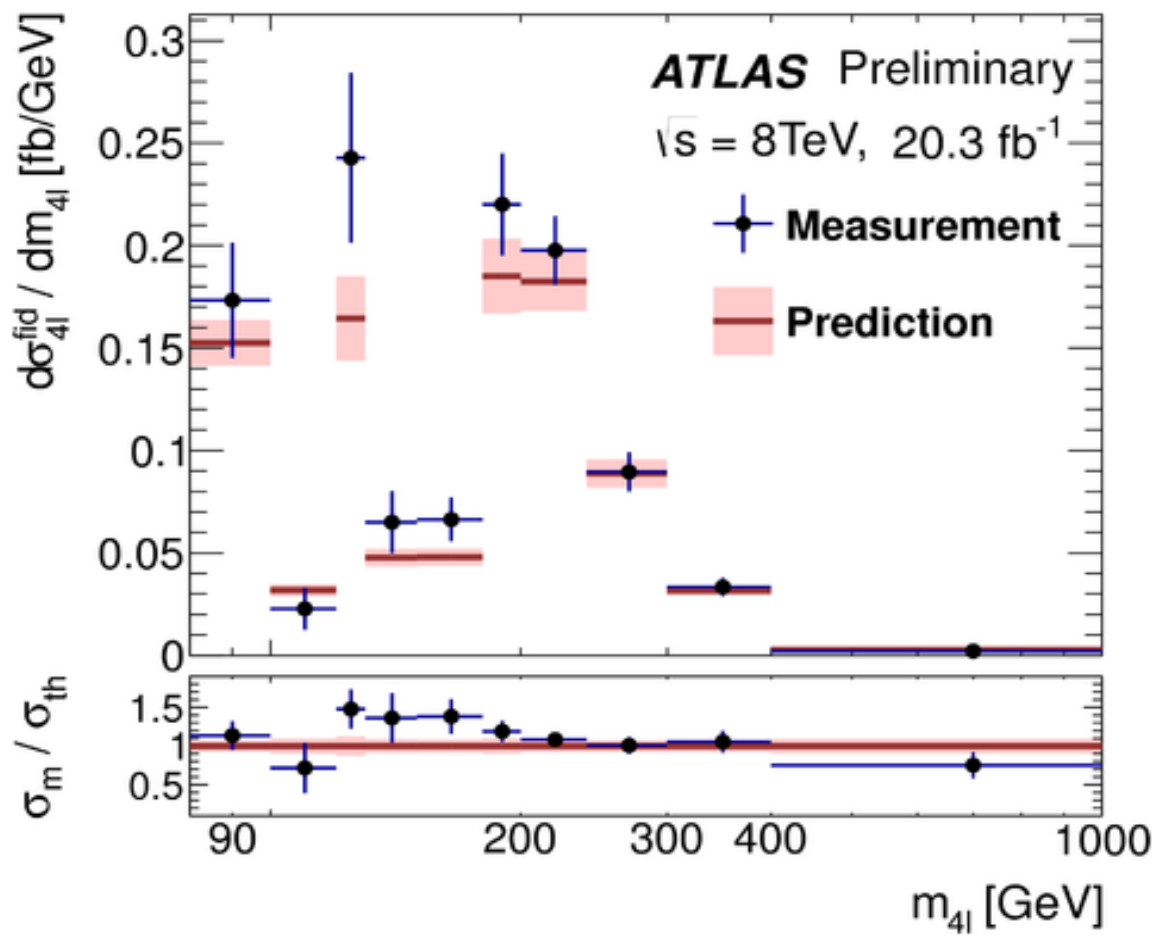




\section{1 inclusive}

Cross Section in the extended phase space [fb] SM Prediction [fb]

\begin{tabular}{lcc}
\hline \hline & Cross Section in the extended phase space [fb] & SM Prediction [fb] \\
\hline$\sigma_{4 e}^{e x t}$ & $17.8_{-2.0}^{+2.1}$ (stat.) ${ }_{-1.1}^{+1.5}$ (syst.) ${ }_{-0.5}^{+0.5}$ (lumi.) & $16.4 \pm 1.4$ \\
$\sigma_{4 \mu}^{e x t}$ & $17.3_{-1.4}^{+1.5}$ (stat.) ${ }_{-0.7}^{+0.9}$ (syst.) ${ }_{-0.5}^{+0.5}$ (lumi.) & $16.4 \pm 1.4$ \\
\hline Combined $\sigma_{4 e+4 \mu}^{e x t}$ & $35.0_{-2.3}^{+2.4}$ (stat.) ${ }_{-1.5}^{+1.9}$ (syst.) ${ }_{-1.0}^{+1.1}$ (lumi.) & $32.9 \pm 1.9$ \\
\hline$\sigma_{2 e 2 \mu}^{e x t}$ & $37.7_{-2.6}^{+2.7}$ (stat.) ${ }_{-2.0}^{+2.5}$ (syst.) ${ }_{-1.1}^{+1.1}$ (lumi.) & $32.1 \pm 2.0$ \\
\hline \hline Combined $\sigma_{4 \ell}^{e x t}$ & $73_{-4}^{+4}$ (stat.) ${ }_{-4}^{+4}$ (syst.) ${ }_{-2}^{+2}$ (lumi.) & $65 \pm 4$ \\
\hline
\end{tabular}

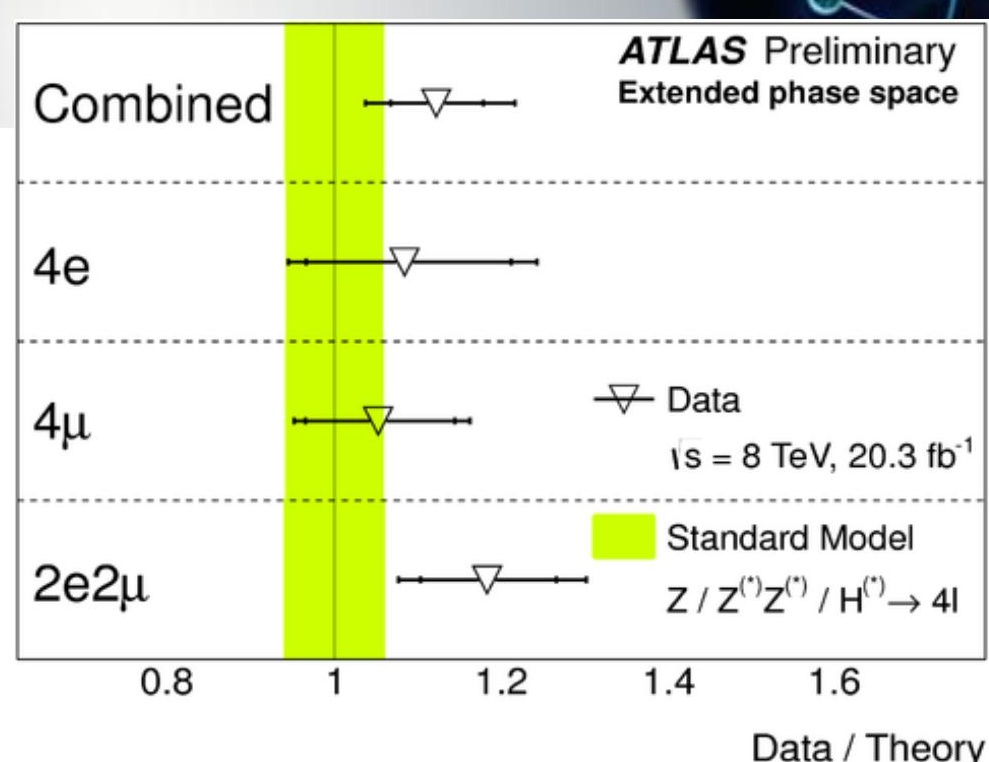

We interpret discrepancies between signal strength and current LO prediction as missing higher order correction to gg->4l: $\mu_{\mathrm{gg}}=2.4 \pm 1.0$ (stat.) \pm 0.5 (syst.) \pm 0.8 (theory)

Inclusive gg component is extracted from data in $\mathrm{m}(4 \mathrm{I})>180 \mathrm{GeV}$ region

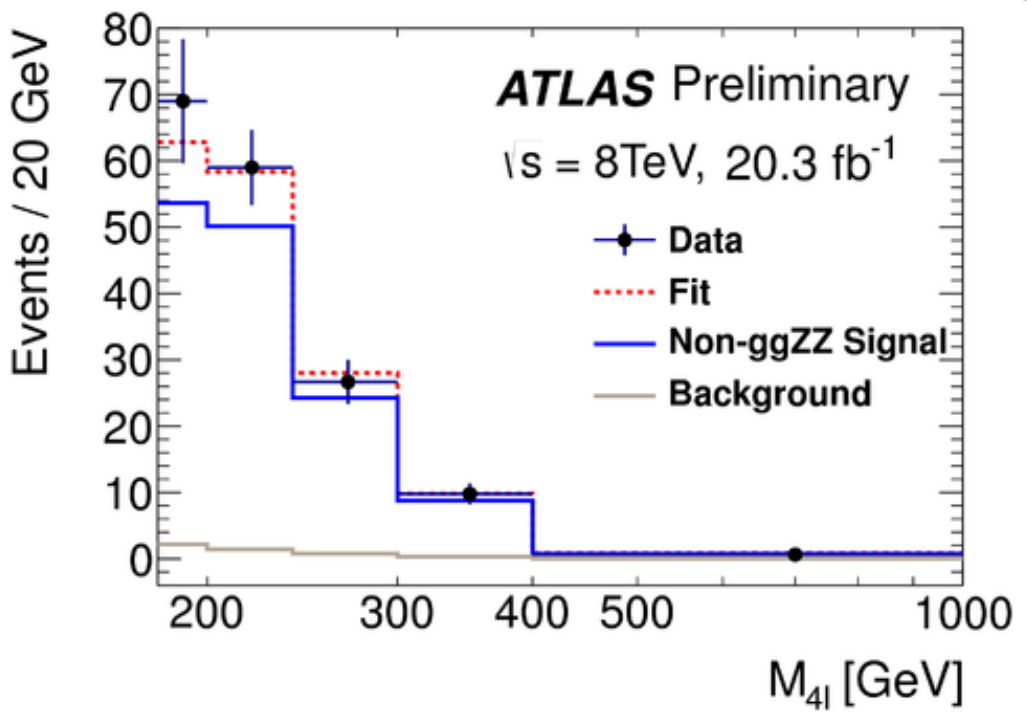


Vector boson fusion Z

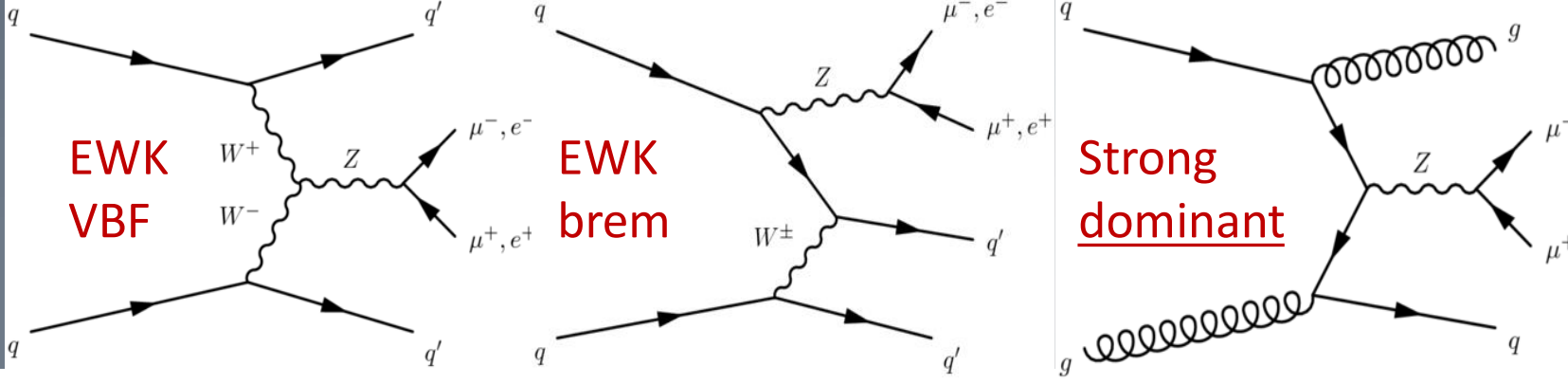

arXiv:1401.7610

JHEP04(2014)031

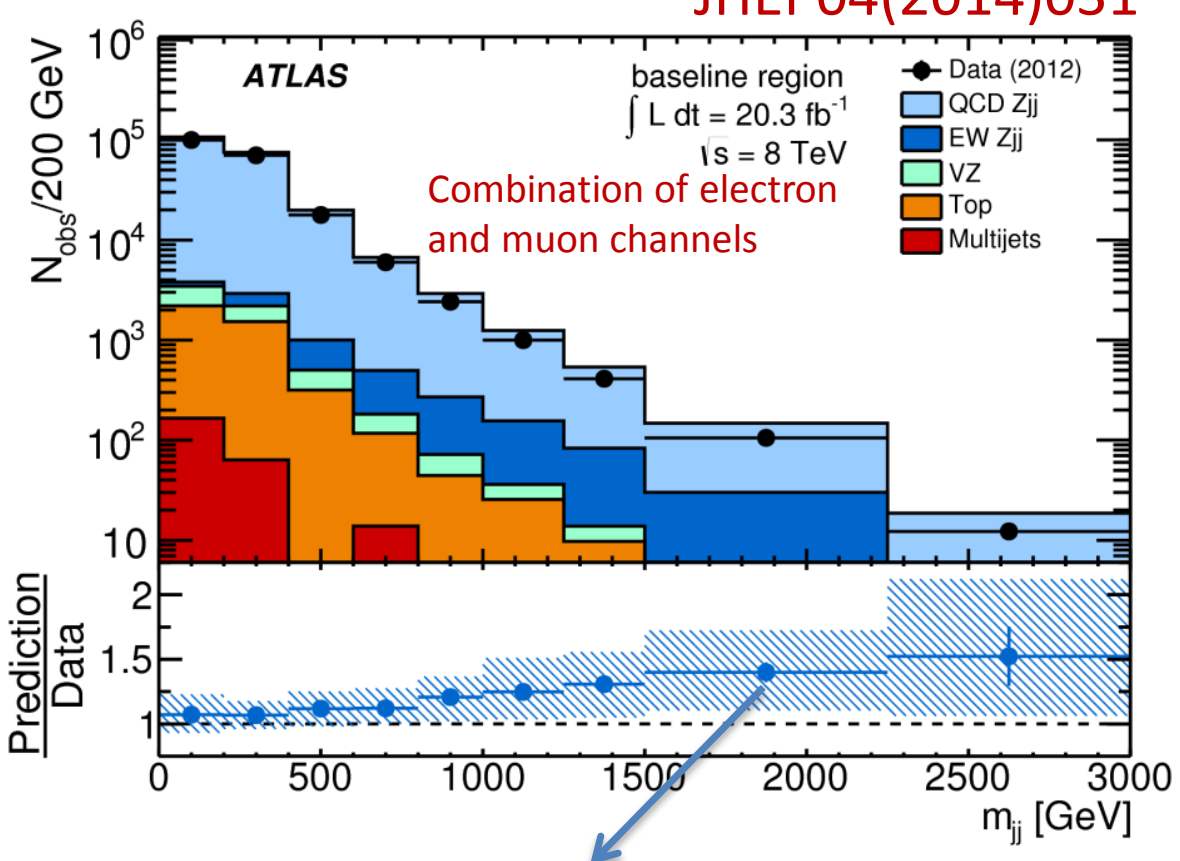

Generator mismodelling at high $\mathrm{mjj}$

\section{Selection}

production which and needs to be correctly evaluated before aTGC (WWZ) measurements

\section{Signature EWK process}

$>$ harder mjj and rapidity gap DeltaYjj distributions

$>$ better balance pT of the system Zjj than strong production

$>$ opposite-sign same-flavor lepton pair within $81 \mathrm{GeV}<\mathrm{mZ}<101 \mathrm{GeV}$

$>\geq 2$ high pT jets search region: $\mathrm{mjj}>250 \mathrm{GeV}$ and central jet veto 


\section{Vector boson}

fusion Z

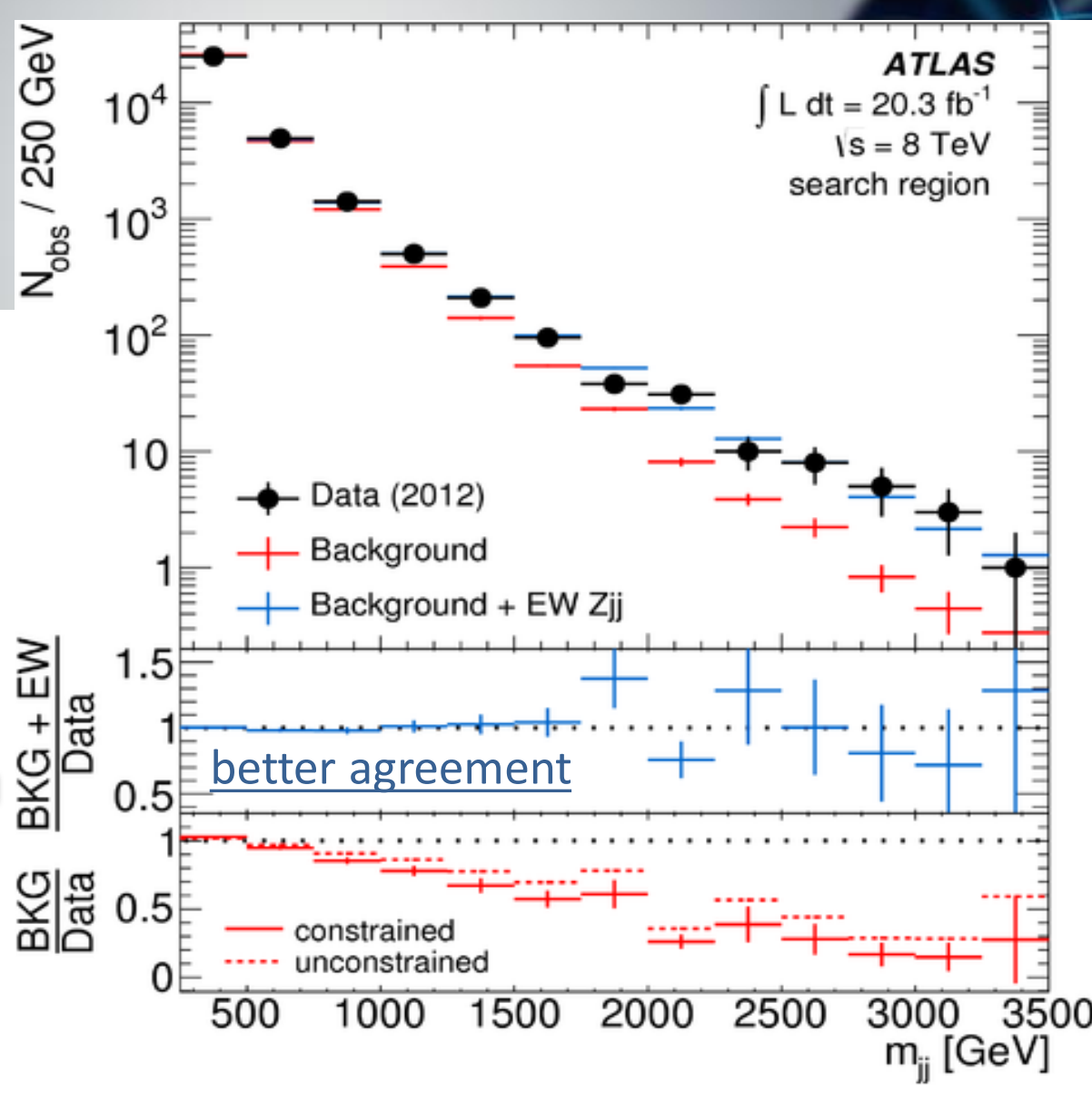

Results

$>$ background-only hypothesis rejected with significance above $\mathbf{5 \sigma}$

$>$ good agreement with Powheg prediction

Fiducial cross section:

$\sigma_{\mathrm{EWK}}($ measured $)=54.7 \pm 11.5 \mathrm{fb}$ $\sigma_{\mathrm{SM}}($ Powheg $)=46.1 \pm 1.0 \mathrm{fb}$
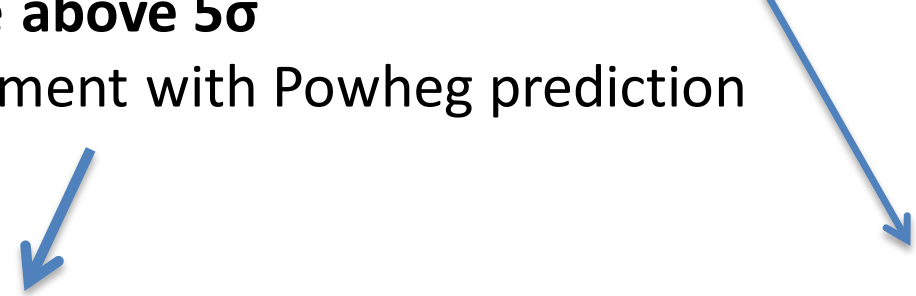

\begin{tabular}{|lcccc|}
\hline aTGC & $\Lambda=6 \mathrm{TeV}($ obs $)$ & $\Lambda=6 \mathrm{TeV}(\exp )$ & $\Lambda=\infty($ obs $)$ & $\Lambda=\infty(\exp )$ \\
\hline$\Delta g_{1, Z}$ & {$[-0.65,0.33]$} & {$[-0.58,0.27]$} & {$[-0.50,0.26]$} & {$[-0.45,0.22]$} \\
$\lambda_{Z}$ & {$[-0.22,0.19]$} & {$[-0.19,0.16]$} & {$[-0.15,0.13]$} & {$[-0.14,0.11]$} \\
\hline
\end{tabular}

aTGC

- different from WZ (space-like vs timelike propag.)

- search region + cut on mjj> 1TeV

- Modified by a dipole form factor, depending on unitarization scale $\Lambda$ 
Vector boson scattering WW
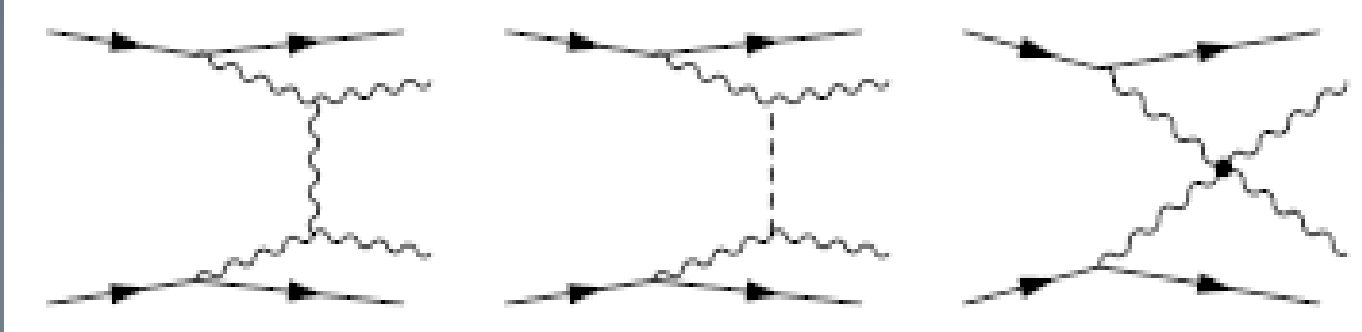

arXiv:1405.6241

Phys. Rev. Lett. 113, 141803 (2014)

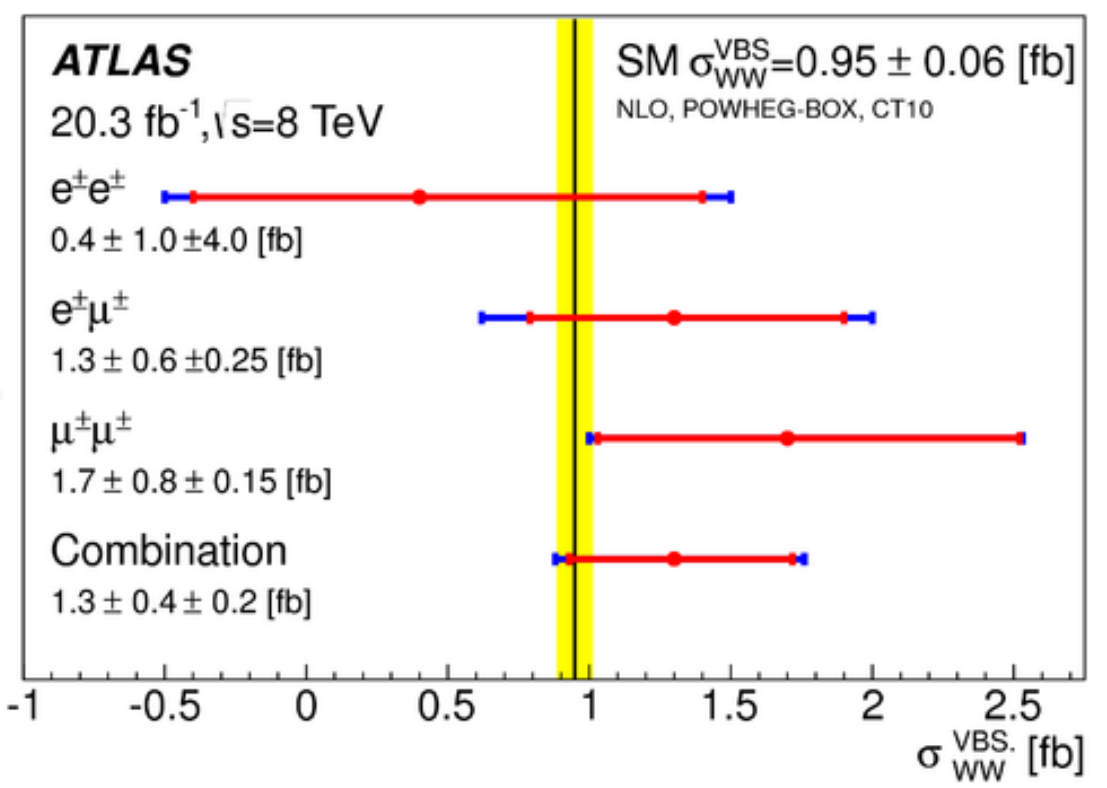

VBS region is used to set first anomalous quartic gauge coupling (aQGC) limits on parameters: $\alpha 4, \alpha 5$
ATLAS

$20.3 \mathrm{fb}^{-1}, 1 \mathrm{~s}=8 \mathrm{TeV}$ $\mathrm{pp} \rightarrow \mathrm{W}^{ \pm} \mathrm{W}^{ \pm} \mathrm{ij}$

$\mathrm{K}$-matrix unitarization

confidence intervals

$-0.4=\square 68 \% \mathrm{CL}$

$-0.6=-$ expected $95 \% \mathrm{CL}$

$\times$ Standard Model

$\begin{array}{lllllllll}-0.4 & -0.3 & -0.2 & -0.1 & 0 & 0.1 & 0.2 & 0.3 & 0.4\end{array}$ ults)

th theory prediction

8

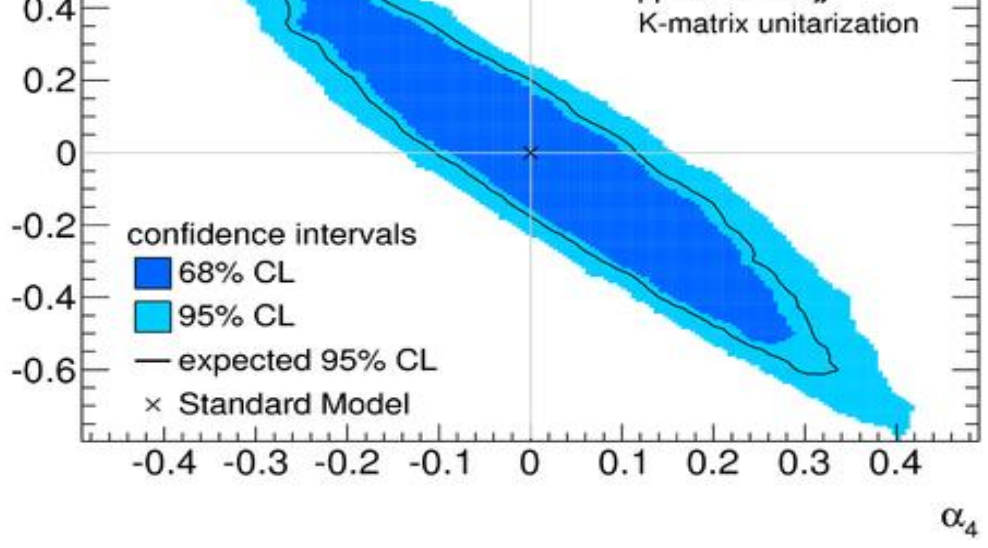




\section{WYY production}

\section{Cross sections}

- Higher by $1.9 \sigma$ than SM NLO prediction in inclusive case

- Better agreement in exclusive case

\begin{tabular}{|c|c|c|}
\hline & $\sigma^{\text {fid }[\mathrm{fb}]}$ & $\sigma^{\mathrm{MCFM}}[\mathrm{fb}]$ \\
\hline \multicolumn{3}{|c|}{ Inclusive $\left(N_{\text {jet }} \geq 0\right)$} \\
\hline $\begin{array}{l}\mu \nu \gamma \gamma \\
e v \gamma \gamma \\
\ell v \gamma \gamma\end{array}$ & $\begin{array}{c}7.1_{-1.2}^{+1.3} \text { (stat.) } \pm 1.5 \text { (syst.) } \pm 0.2 \text { (lumi.) } \\
4.3_{-1.6}^{+1.8} \text { (stat.) }{ }_{-1.8}^{+1.9} \text { (syst.) } \pm 0.2 \text { (lumi.) } \\
6.1_{-1.1}^{+1.1} \text { (stat.) } \pm 1.2 \text { (syst.) } \pm 0.2 \text { (lumi.) }\end{array}$ & $2.90 \pm 0.16$ \\
\hline \multicolumn{3}{|c|}{ Exclusive $\left(N_{\text {jet }}=0\right)$} \\
\hline $\begin{array}{l}\mu \nu \gamma \gamma \\
e v \gamma \gamma \\
\ell v \gamma \gamma\end{array}$ & $\begin{array}{c}3.5 \pm 0.9 \text { (stat.) }{ }_{-1.0}^{+1.1} \text { (syst.) } \pm 0.1 \text { (lumi.) } \\
1.9_{-1.1}^{+1.4} \text { (stat.) } \\
2.9_{-0.1}^{+0.1} \text { (syst.) } \pm 0.1 \text { (lumi.) } \\
-1.0 \\
\end{array}$ & $1.88 \pm 0.20$ \\
\hline
\end{tabular}

\section{Anomalous quartic couplings limits are}

set for exclusive case

- $\mathrm{M}_{\nu \gamma}>300 \mathrm{GeV}$

- Limits better or similar to LEP and DO

$a_{C}{ }^{W} / \Lambda^{2}=-2 m_{W}{ }^{2}\left(g^{-2}+g^{\prime-2}\right) g^{\prime}{ }^{2}{ }_{M 3} / \Lambda^{4}$

$a_{0}{ }^{W} / \Lambda^{2}=2 m_{W^{2}}\left(g^{-2}+g^{\prime-2}\right) g^{2} f_{M 2} / \Lambda^{4}$,

$\mathrm{f}_{\mathrm{TO}}{ }^{\mathrm{E}} / \Lambda^{4}=\mathrm{g}^{4} \mathrm{f}_{\text {TO }} / \Lambda^{4}$

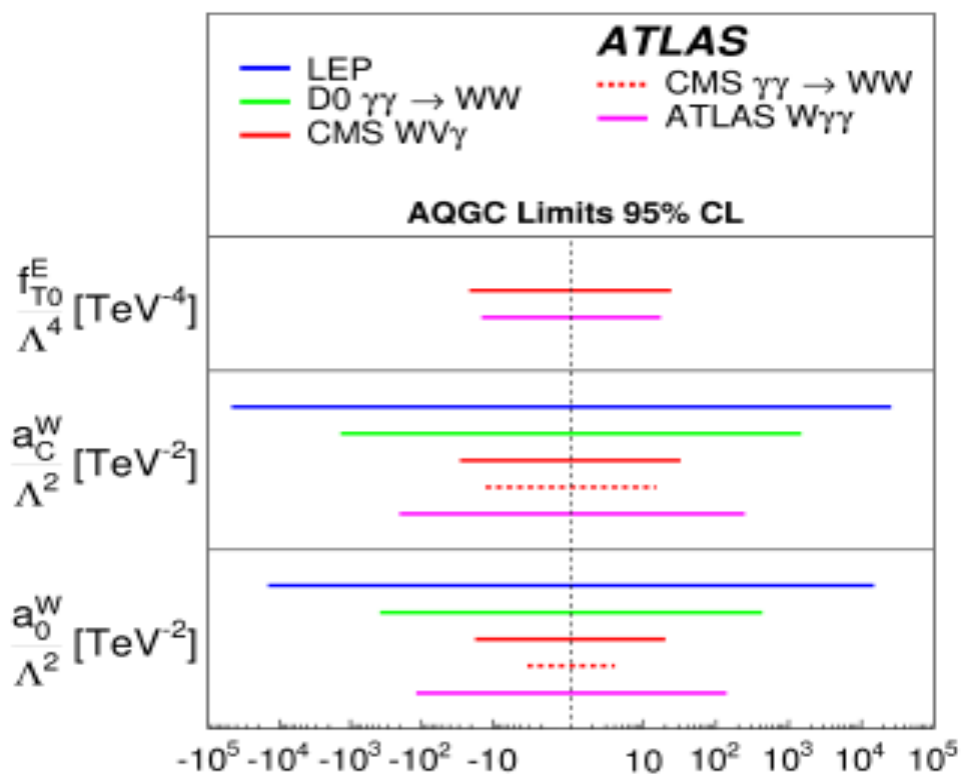




\section{BONUS: Run 2 meas}

ATL-PHYS-PUB-2015-021
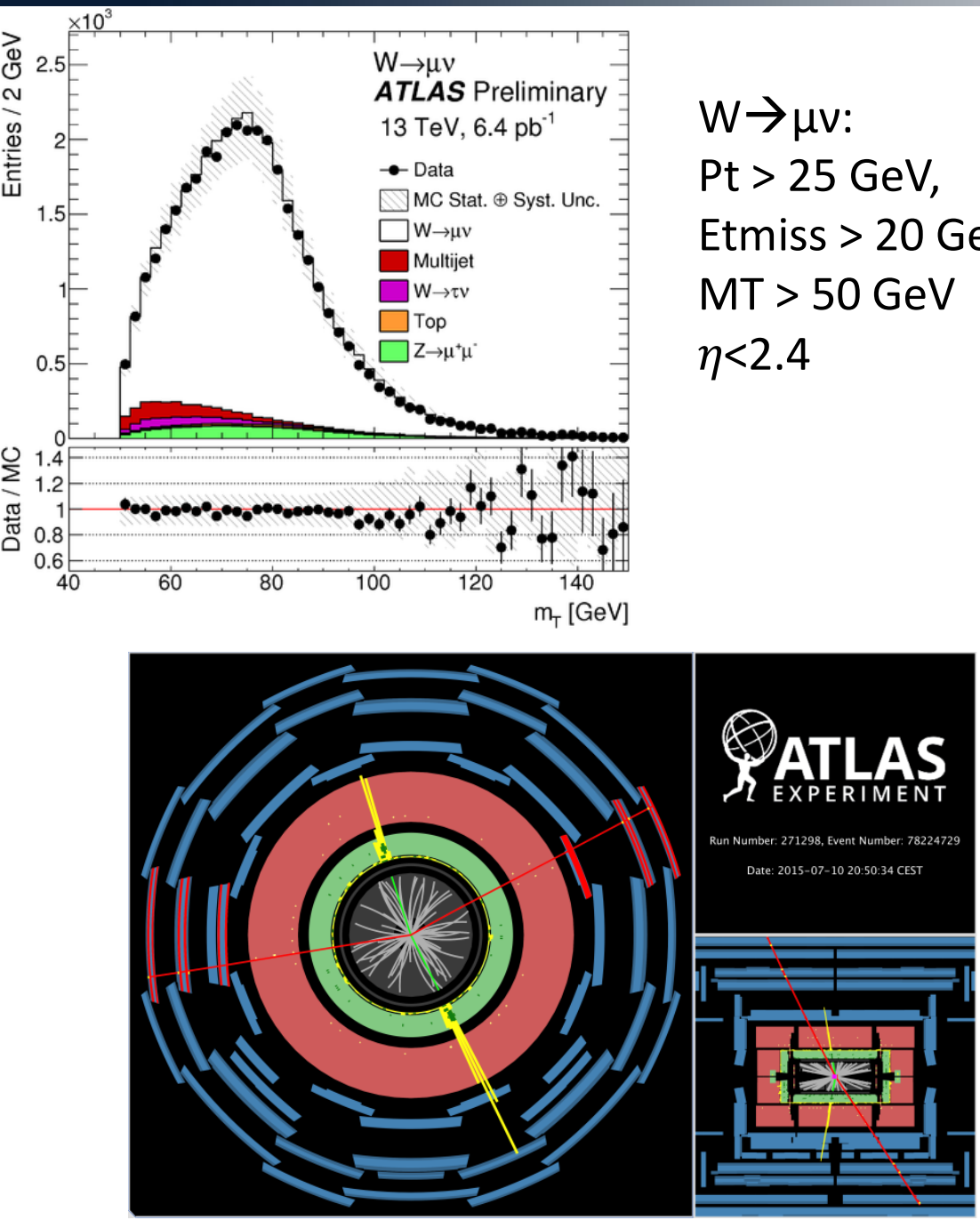

Reasonable agreement with MC

$W \rightarrow \mu v:$

Pt $>25 \mathrm{GeV}$, Etmiss $>20 \mathrm{GeV}$, $\mathrm{MT}>50 \mathrm{GeV}$ $\eta<2.4$

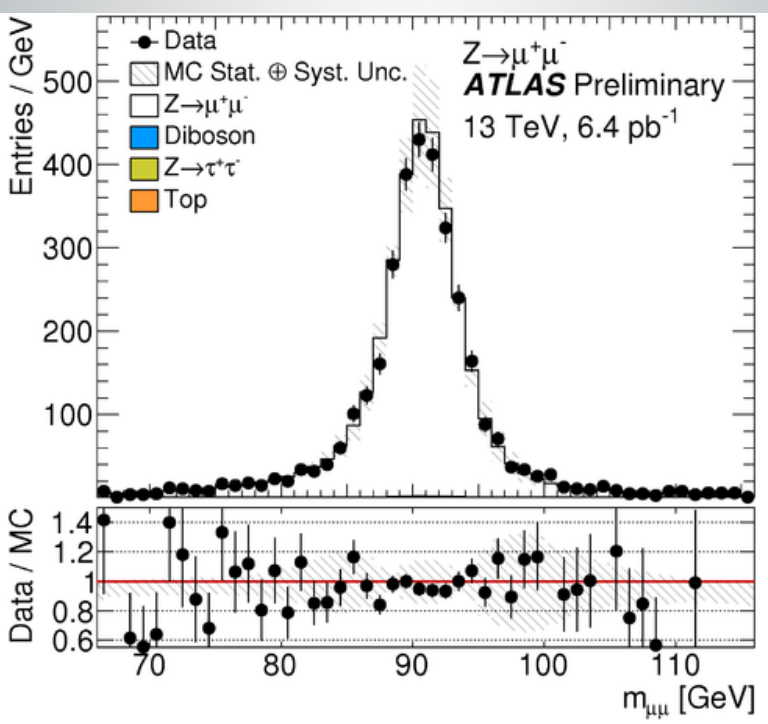

$Z \rightarrow \mu \mu:$

Pt $>25 \mathrm{GeV}$, $66<\mathrm{M}(\mathrm{II})<116 \mathrm{GeV}$ $\eta<2.4$
Display of a $Z(e e) Z(\mu \mu)$ candidate event from proton-proton collisions recorded by ATLAS, with LHC beams at a collision energy of $13 \mathrm{TeV}$. 


\section{Summary}

The measurements presented in this talk provide important tests of electroweak sector in the Standard model (SM) and probes of new physics:

$>$ Measurements of WW production

$>4 \mathrm{l}$ production and strength of the gluon-gluon fusion component

$>$ Electroweak production of $\mathrm{Zjj}$, sensitive to vector boson fusion and limits on aTGCs

$>$ First evidence of vector boson scattering and limits on aQGCs

$>$ First measurement of triboson ( $W \gamma \gamma$ ) production in ATLAS and limits on aQGCs

ATLAS public results are available here:

https://twiki.cern.ch/twiki/bin/view/AtlasPublic/StandardModelPublicResults

More electroweak results with $8 \mathrm{TeV}$ will come soon! 


\section{The end. Thank you!}




\section{Back up slides}




\section{exclusive di-lepton}

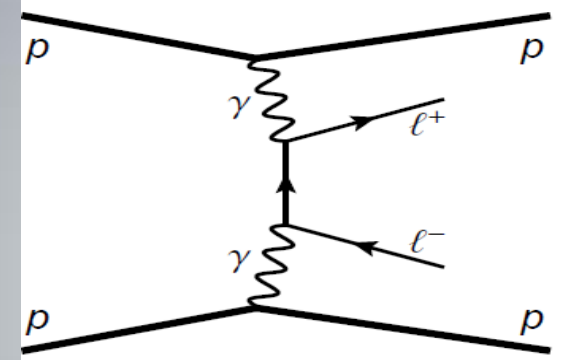

The cross-section of the $\mathrm{pp}(\nu \gamma) \rightarrow \mathrm{I}+\mathrm{I}-\mathrm{X}$ process has been predicted to increase with energy and constitutes a non-negligible background to Drell-Yan reactions.

$>$ Can be calculated in the framework of quantum electrodynamics (QED)

$>$ Preselection

- exactly 2 back-to-back leptons

- $\mathrm{Z}$ region removed

- binned maximum-likelihood fit to the measured dilepton acoplanarity distribution

$>$ Exclusive selection

- $3 \mathrm{~mm}$ dilepton-vertex longitudinal isolation

- pT of the dilepton system $<1.5 \mathrm{GeV}$

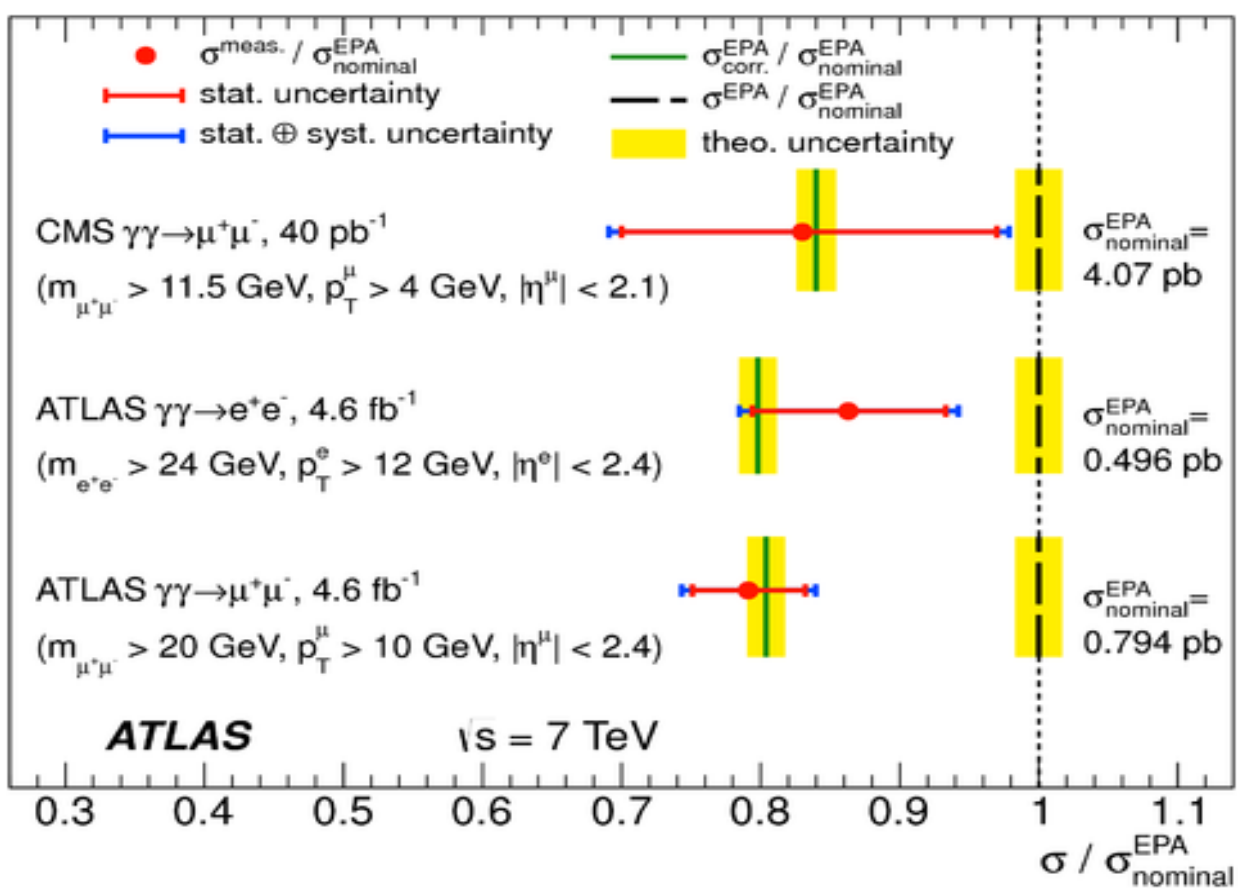

Agreement with similar CMS measurement 20\% below the nominal EPA prediction, and consistent with the suppression expected due to proton absorption contributions. 
